\title{
Editorial
}

\section{Nanotechnologies for Biosensor and Biochip}

\author{
Moon Il Kim, ${ }^{1}$ Tae Jung Park, ${ }^{2}$ Elena E. Paskaleva, ${ }^{3}$ Fangfang Sun, ${ }^{4}$ \\ Jin W. Seo, ${ }^{5}$ and Krunal K. Mehta ${ }^{6}$ \\ ${ }^{1}$ Department of BioNano Technology, Gachon University, 1342 Seongnamdae-ro, Sujeong-gu, \\ Seongnam, Gyeonggi 461-701, Republic of Korea \\ ${ }^{2}$ Department of Chemistry, Chung-Ang University, 84 Heukseok-ro, Dongjak-gu, Seoul 06974, Republic of Korea \\ ${ }^{3}$ Center for Biotechnology and Interdisciplinary Studies, Rensselaer Polytechnic Institute, Troy, NY 12180, USA \\ ${ }^{4}$ College of Life Information Science and Instrument Engineering, Hangzhou Dianzi University, Xiasha, \\ Hangzhou, Zhejiang 310018, China \\ ${ }^{5}$ Departement Materiaalkunde (MTM), KU Leuven, Kasteelpark Arenberg 44, Bus 2450, 3001 Leuven, Belgium \\ ${ }^{6}$ Pivotal Purification Process Development, Amgen, Inc., Cambridge, MA 02142, USA
}

Correspondence should be addressed to Moon Il Kim; moonil@gachon.ac.kr

Received 3 December 2015; Accepted 3 December 2015

Copyright (C) 2015 Moon Il Kim et al. This is an open access article distributed under the Creative Commons Attribution License, which permits unrestricted use, distribution, and reproduction in any medium, provided the original work is properly cited.

The biosensing devices are characterized by their biological receptors, which have specificity to their corresponding analytes. These analytes are a vast and diverse group of biological molecules, DNAs, proteins (such as antibodies), fatty acids, or entire biological systems, such as pathogenic bacteria, viruses, cancerous cells, or other living organisms. A main challenge in the development of biosensor applications is the efficient recognition of a biological signal in a low signal-to-noise ratio environment, and its transduction into an electrochemical, optical, or other signals. The advent of nanomaterial technology greatly increased the potential for achieving exquisite sensitivity of such devises, due to the innate high surface-to-volume ratio and high reactivity of the nanomaterial. The second major challenge facing the biosensor application, that of scalability, is addressed by multiplexing and miniaturizing of the biosensor devises into a biochip. In recent years, biosensor and biochip technologies have made significant progress by taking advantages of diverse kinds of nanomaterials that are derived from nanotechnology [1-3].

These materials exhibit unique optical, electronic, magnetic, and catalytic properties, which can be essentially utilized to develop novel biosensors that yield highly enhanced sensitivity, selectivity, and other attributes. Furthermore, the newly developed nanomaterials provide a particularly useful platform for the development of biochips that are ideally suited to meet the demands of biomolecules to facilitate various kinds of biological events. In this special issue, to provide chemists, biochemists, material engineers, and bioengineers with a perspective on the current state-of-the-art of this emerging nanobiotechnological research field, several excellent research results and a comprehensive review on nanotechnologies for biosensor and biochip were reported.

The paper by Y. M. Bae et al. is focused on the applicability of localized surface plasmon resonance (LSPR) substrate with gold nanoparticle array to detect relevant biomarkers. In this work, the LSPR substrate was first fabricated with a lift-off process and its LSPR phenomenon was confirmed by measuring the optical transmission level of the substrate. Then, the antibodies with a high affinity toward target molecules were immobilized on the gold nanoparticle array. The immobilization was confirmed by observing the shift of LSPR peak of the resultant substrate. These new LSPR substrates conjugated with antibodies were successfully applied to detect lowand high-density lipoproteins, which are biomarkers for diagnosing and monitoring of cardiovascular disease.

The colorimetric activity of magnetic nanoparticles (MNPs) was employed to develop a unique biosensor. J. Y. Park et al. reported a colorimetric biosensor based on the peroxidase-like activity of magnetic nanoparticles and DNA 
aptamers having a high affinity toward a target food pathogen Salmonella typhimurium. In this assay system, MNPs were first incubated with aptamers that specifically interact with the target bacterial cells, reducing the peroxidase activity of the MNPs through DNA-mediated shielding of the catalytic activity. After the addition of target Salmonella cells into the solution, specific aptamers on the MNPs interact with the Salmonella, consequently enhancing the peroxidase activity of the MNPs. Overall, the presence and quantity of target Salmonella cells were successfully detected by the colorimetric response produced from the peroxidase-like activity of MNPs. Based on the results, the authors propose that this label-free colorimetric biosensor would be invaluable for detecting DNA-DNA, DNA-protein, DNA-cell, and DNAligand interactions due to its ease of use, low cost, capability to detect with naked eye, and the high stability of MNPs.

C. S. Park et al. investigated the cytotoxicity, particularly autophagy, in RAW264.7 cells exposed to graphene oxide (GO) and its derivatives including dodecylamine$\mathrm{GO}$, reduced GO, and sodium dodecyl sulfate-reduced GO. They showed that all the GO types exerted cytotoxic effects on RAW264.7 cells in a concentration-dependent manner. Higher concentrations of the GO types downregulated the expression of PU.1, a unique transcription factor in monocytes and macrophages, and decreased the conversion of LC3A/B-I to LC3A/B-II, suggesting that PU.1 was associated with autophagy in RAW264.7 cells. These results suggest that surface-functionalized GOs exert cytotoxic effects in a concentration-dependent manner by changing the expression of critical genes and inducing autophagy in macrophages.

A binary immiscible polymer blended system with high stability is also reported by J.-H. Kim et al. In this article, domain structures of spin-coated immiscible poly(methyl methacrylate) (PMMA) and ultraviolet curable poly(urethane acrylate) (PUA) blends were studied using atomic force microscopy (AFM). Since the cross-linked PUA in the polymer-blended films provided strong chemical stability in various solvents, target materials for dissolution could be selectively available via a simple curing process. In addition, morphology of the PMMA/PUA blends, including domain size, height, and nanoscale features, could be easily controlled by changing composition of the blends. Based on the results, the authors suggest potentials for various applications related to nanotextured surfaces and soft lithography.

The electronic and molecular structure of doped graphene was theoretically investigated with solid-state density functional calculations. Y. H. Hwang et al. studied the graphene-organic molecule complex, referred to as organic doping in material science community. With the density functional calculations, the authors determined the role of amine-based aromatic compounds in graphene doping, binding to graphene through long-range interactions such as $\pi-\pi$ interactions and $\mathrm{C}-\mathrm{H} \cdots \pi$ hydrogen bonding. The electronic structures of pristine graphene were compared to that of doped graphene to help understand the electronic structure of the material at molecular level. In addition to the investigation of the molecular interactions, the paper showed that screening of organic molecules would benefit from a sold-state density functional calculation to predict the experimental results from doping or sensing organic molecules with graphene.

A comprehensive review on recent nanozyme (nanomaterial-based artificial enzymes) research is presented by $\mathrm{H}$. Y. Shin et al. It covers the fundamentals and the applications for development of novel biosensors, immunoassays, cancer diagnostics, and therapeutics, as well as environmental engineering technologies. The review concludes with discussion on the current challenges and future prospects of using nanozymes in biotechnology.

We hope that this special issue will provide new insights and research motivation to the interested readers for further advancement in biosensor and biochip applications through state-of-the-art nanotechnology.

\section{Acknowledgment}

We would like to thank all authors for their valuable contributions to this special issue. We also would like to acknowledge the referees for their indispensable efforts to improve the quality of this special issue.

\author{
Moon Il Kim \\ Tae Jung Park \\ Elena E. Paskaleva \\ Fangfang Sun \\ Jin W. Seo \\ Krunal K. Mehta
}

\section{References}

[1] Y. Song, W. Wei, and X. Qu, "Colorimetric biosensing using smart materials," Advanced Materials, vol. 23, no. 37, pp. 42154236, 2011.

[2] H. Wei and E. Wang, "Nanomaterials with enzyme-like characteristics (nanozymes): next-generation artificial enzymes," Chemical Society Reviews, vol. 42, no. 14, pp. 6060-6093, 2013.

[3] Y. Xia, Y. Xiong, B. Lim, and S. E. Skrabalak, "Shape-controlled synthesis of metal nanocrystals: simple chemistry meets complex physics?" Angewandte Chemie-International Edition, vol. 48, no. 1, pp. 60-103, 2009. 

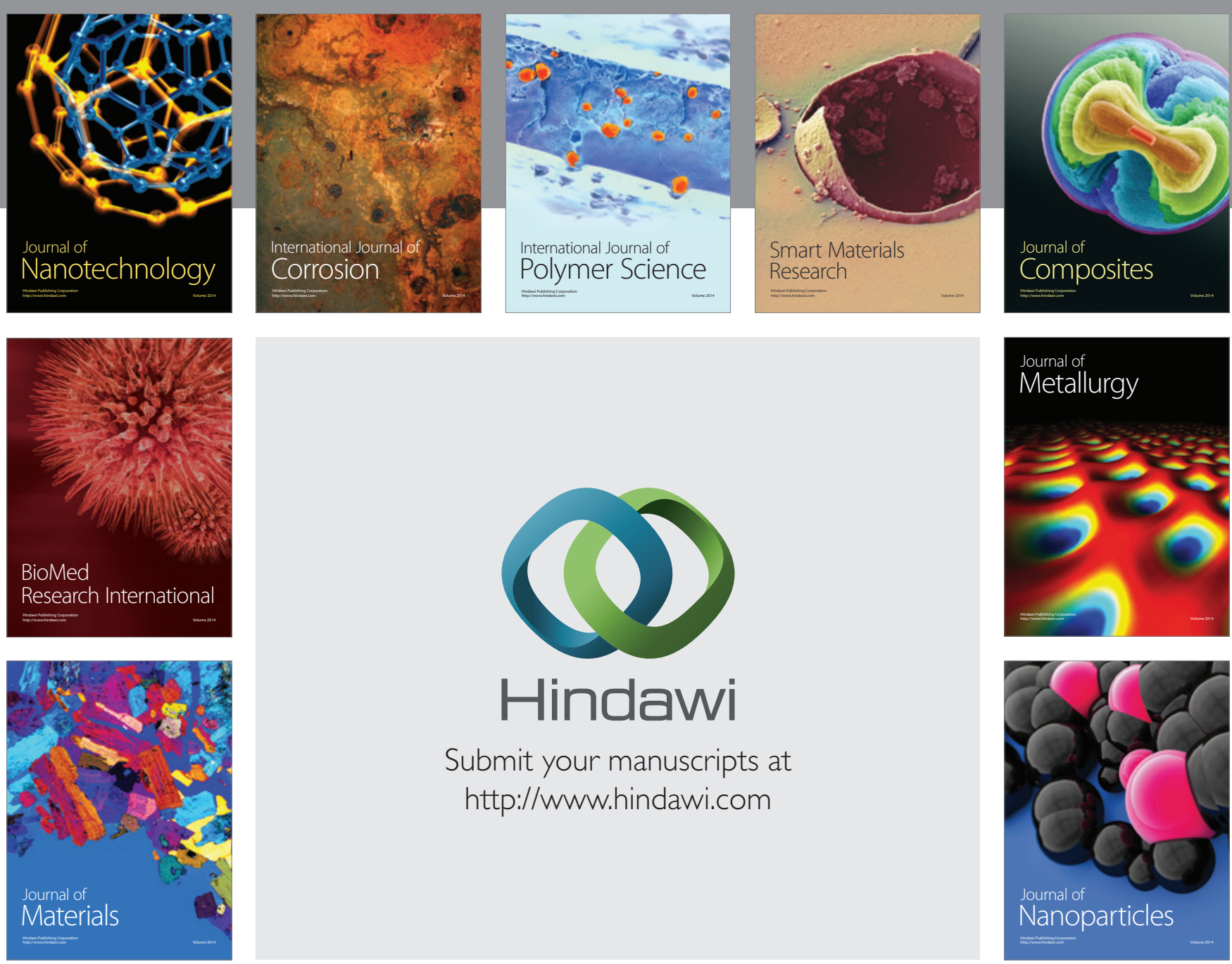

Submit your manuscripts at http://www.hindawi.com
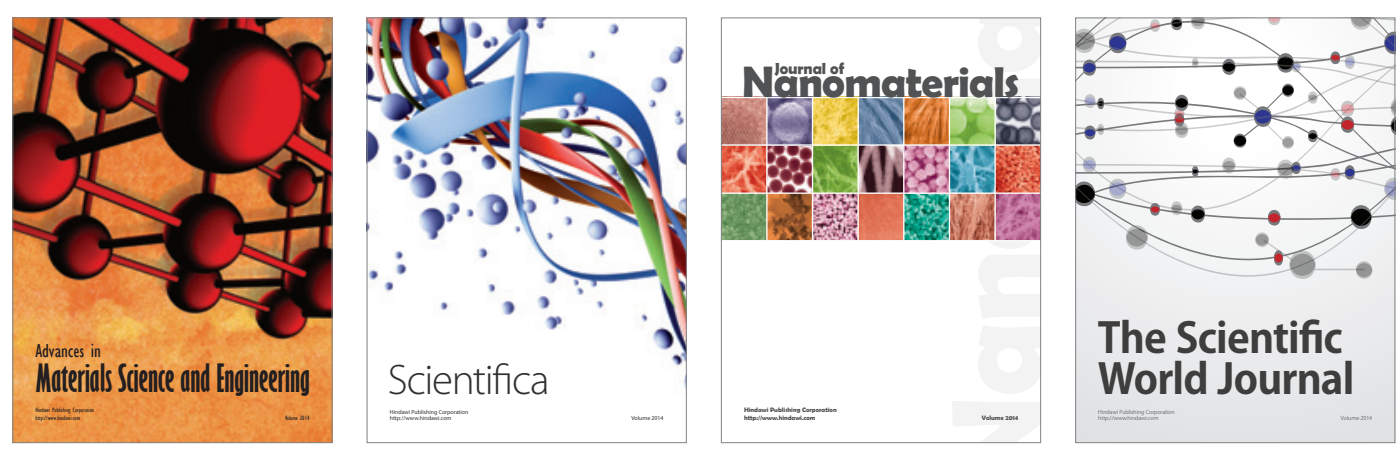

\section{The Scientific World Journal}
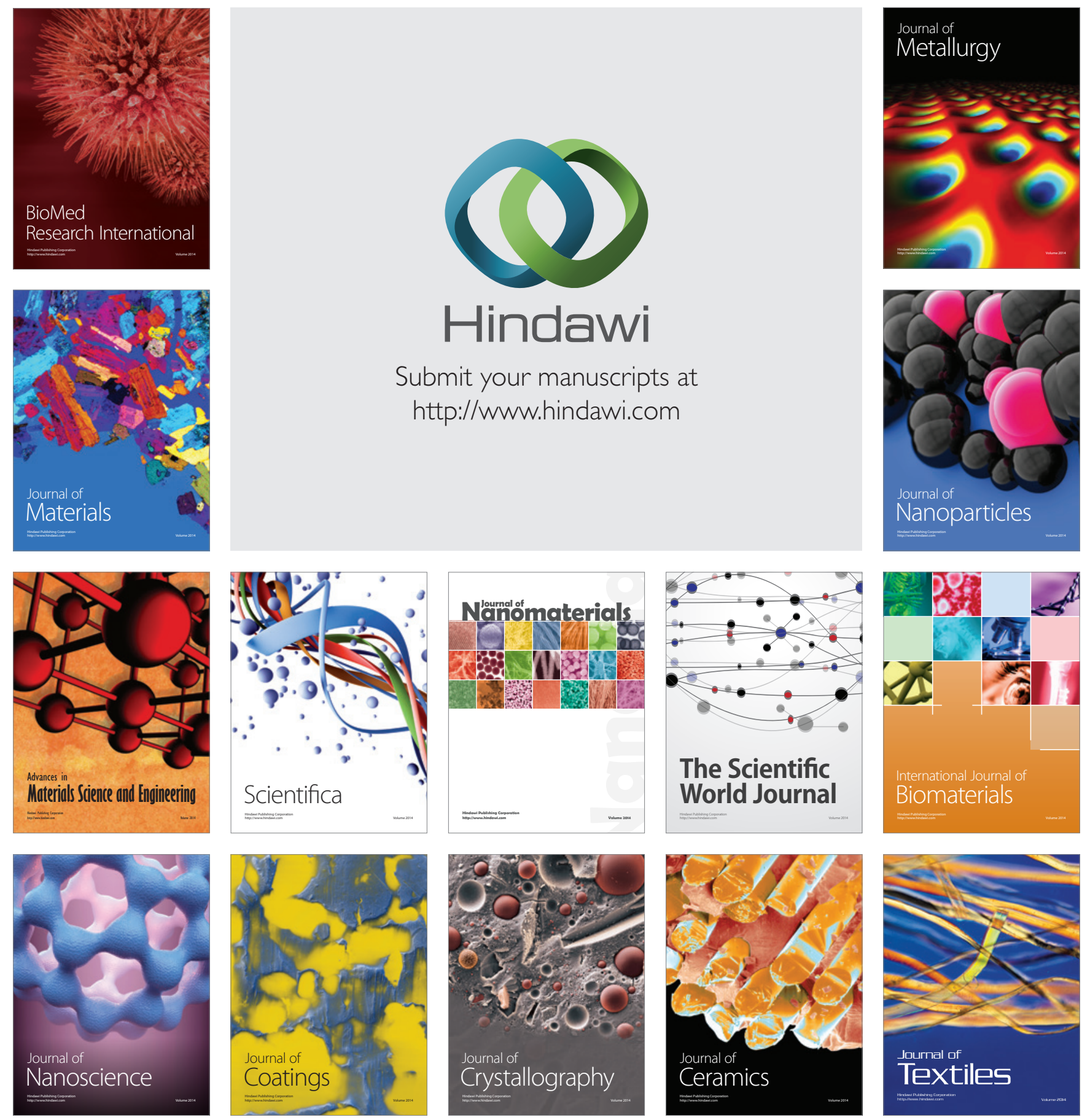